\title{
Adult adrenal neuroblastoma: A case report
}

\author{
SHENGHAN XU ${ }^{1 *}$, WENJUN ZHANG ${ }^{1 *}$, BANGWEI CHE $^{1 *}$, JINJUAN ZHANG $^{1}$, JUN HE $^{1}$, \\ DONGDONG LIU $^{1}$, PAN CHEN ${ }^{1}$, YI MU ${ }^{1}$, KEHANG CHEN ${ }^{1}$ and KAIFA TANG ${ }^{1,2}$ \\ ${ }^{1}$ Department of Urology, Affiliated Hospital of Guizhou Medical University; \\ ${ }^{2}$ Institute of Medical Science of Guizhou Medical University, Guiyang, Guizhou 550004, P.R. China
}

Received March 5, 2021; Accepted July 14, 2021

DOI: $10.3892 / \mathrm{mco} .2021 .2378$

\begin{abstract}
Adrenal neuroblastoma (NB) is very rare in adults. According to the literature, $<100$ cases have been reported worldwide to date, with $>90 \%$ of the patients aged $<10$ years. As the early symptoms of the disease are not obvious, distant metastasis has often already occurred when the patients develop clinical symptoms. This lack of obvious symptoms may lead to misdiagnosis and inadequate treatment. Imaging and laboratory examinations are crucial for the diagnosis of NB, but reaching a definitive diagnosis prior to surgery is challenging, as the final diagnosis ultimately depends on histopathological examination. The aim of the present study was to report the rare case of a 40-year-old woman with adrenal left NB who underwent tumor resection. No tumor recurrence was observed at the 3-month and 1-year postoperative follow-up, but a repeat computed tomography at the 3-year postoperative follow-up indicated metastases; the patient refused further treatment and eventually succumbed to the disease within 1 month. The aim of the present case was to emphasize the importance of individualized therapy and long-term, close follow-up of the patients. The clinical characteristics and treatment of this case of adrenal NB were also summarized and analyzed in order to raise clinical awareness of this rare disease.
\end{abstract}

\section{Introduction}

Adrenal neuroblastoma (NB) is a tumor of the autonomic nervous system originating from primordial neural crest cells. The occurrence of NB is common in children but very rare in adults, and the clinical manifestations are non-specific (1). NB is highly malignant, usually characterized by invasive growth and propensity for organ metastasis. Compared with

Correspondence to: Professor Kaifa Tang, Department of Urology, Affiliated Hospital of Guizhou Medical University, 9 Beijing Road, Guiyang, Guizhou 550004, P.R. China

E-mail: tangkaifa@gmc.edu.cn

${ }^{*}$ Contributed equally

Key words: neuroblastoma, adrenal gland neoplasms, neural crest, adult, immunohistochemistry pediatric patients, the prognosis of adult patients with NB is poor (2). The most common primary site in adult patients (aged 18-60 years) is the central nervous system (39\%), followed by the retroperitoneum (17\%). The most common manifestations of adrenal NB in adults are the presence of an abdominal mass, abdominal pain, or other clinical manifestations caused by tumor growth and metastasis, such as spinal cord compression and hypertension (3).

\section{Case report}

In June 2016, a 40-year-old female patient was admitted to the Affiliated Hospital of Guizhou Medical University (Guiyang, China) with complaints of intermittent lower left back pain and progressive pain in the lower right abdomen for $>3$ months. The patient had a history of nephrolithiasis for 10 years and rheumatoid arthritis for 1 year, for which she received no treatment. On admission, the patient had a height of $160 \mathrm{~cm}$, a weight of $70 \mathrm{~kg}$ and a blood pressure of $127 / 96 \mathrm{mmHg}$. No obvious abnormalities were identified on physical examination. Urine analysis showed hematuria. No abnormality was identified in the remaining laboratory tests. Computed tomography (CT) examination (Fig. 1A and B) revealed the presence of a neoplastic lesion in the left suprarenal gland, sized $\sim 5.3 \times 5.0 \times 4.3 \mathrm{~cm}$. Following discussion with the patient and her family, laparoscopic radical tumor resection was recommended. The surgery was successful. Intraoperatively, the adrenal tumor was found to be surrounded by an adipose tissue capsule and it was mobile. Finally, the tumor was completely removed and sent to the Department of Pathology. The results of the pathological examination of the surgical specimen (Fig. 2A-D) included the following: H\&E staining of the tumor revealed round cells of different sizes, with little cytoplasm, deep staining and diffuse nuclear distribution, as well as a small number of lymphocytes in the fibrous stroma; in addition, the cancer cells were arranged in nest-like solid sheets. Immunohistochemical examination revealed that the tumor cells were positive for synaptophysin, CD56, CD99 and chromogranin A, partially positive for S-100 and Ki-67 (80\%), and negative for other markers, such as leukocyte common antigen, cytokeratin 5/6 and P63.

The final diagnosis was adrenal NB. Due to personal and family-related issues, the patient did not undergo further antitumor therapy, such as radiotherapy or chemotherapy. There were no obvious abnormalities on imaging and laboratory 
examinations at the 3-month postoperative follow-up, and no tumor recurrence was observed on imaging examination at the 1-year postoperative follow-up. However, a repeat CT at the 3-year postoperative follow-up revealed pulmonary metastasis. The patient refused chemotherapy and targeted therapy, and finally succumbed to the disease within 1 month.

\section{Discussion}

Adrenal NB in adults is rare, and imaging plays an important role in the diagnosis, staging, curative effect evaluation and long-term follow-up of patients with NB (4).On CT examination, NB manifests as a round cystic, solid, or irregular solid mass with uneven density, necrotic areas and patchy calcification in the center, with vascular entrapment and vasodilation in or around the tumor (5). The typical manifestations of NB on magnetic resonance imaging (MRI) examination include calcification, necrosis, hemorrhage and cystic degeneration. In a previous study, MRI revealed inhomogeneous signal intensity, low signal intensity on T2-weighted imaging (T2WI) and high signal intensity on T2WI (6). Metaiodobenzylguanidine (MIBG) enters cells through the norepinephrine transporter (7), providing a sensitive and specific method of evaluating cartilage and bone tumors. NBs produce catecholamine metabolites, which can be used for the biological diagnosis of NB. The abnormal level of catecholamines secreted by NB can be detected by examining the levels of plasma epinephrine and norepinephrine (8); However, as this test lacks specificity, it should be limited to cases with unclear symptoms and should not be used for routine screening (9). The definitive diagnosis of NB ultimately depends on histopathological and immunohistochemical examination.

The International Neuroblastoma Risk Group (INRG) staging system (10) has been developed for statistical and clinical risk stratification of different subgroups of patients with NB. The classification is based on age at the time of diagnosis, INRG tumor stage, histological classification, tumor differentiation, DNA ploidy, MYCN oncogene locus and copy number status of chromosome 11q. In the INRG staging system, the degree of local disease is determined by the presence of image-defined risk factors (L1 and L2, respectively), whereas Phase $\mathrm{M}$ is used for widely spread disease. According to the 5-year event-free survival (EFS), Patients with 5-year EFS $>85 \%,>75 \%$ to $\leq 85 \%,>50 \%$ to $\leq 75 \%$, or $<50 \%$ were classified as very low, low, intermediate, or high risk, respectively. The INRG classification system greatly promotes the comparison of risk-based clinical trials conducted in different parts of the world and the development of international collaborative research (10).

There is currently no unified standard for the treatment of adult NB. In the present case, laparoscopic resection of the tumor was performed, but no adjuvant chemotherapy or radiotherapy were administered postoperatively. Laparoscopic surgery has the advantages of less trauma and quick recovery after surgery, which is superior to traditional laparotomy (11). Owing to the rarity of adult NB, there is a lack of chemotherapeutic drugs for standard treatment; however, radiotherapy is recommended if the disease progresses after surgery and chemotherapy. For high-risk patients, active comprehensive treatment, including surgical resection, high-dose chemotherapy combined with stem cell rescue, radiotherapy and biological/immunotherapy can improve patient prognosis (12). In a Memorial-Sloan Kettering retrospective study of 30 patients with advanced $\mathrm{NB},>50 \%$ of the patients were treated with high-dose induction chemotherapy after surgery to achieve optimal results (13). Surgical resection is the main treatment for low-risk patients. Alternative combination chemotherapy includes cyclophosphamide, carboplatin, cisplatin, etoposide, teniposide and doxorubicin (14). Compared with cisplatin alone, the biological coupling of cobalt sulfur nanoparticles and cisplatin led to a $57-78 \%$ reduction in NB cell proliferation (15). It has been demonstrated that the anaplastic lymphoma kinase (ALK) gene is associated with NB susceptibility, and that changes in MYCN gene status may also lead to changes in ALK (16). ALK mutations in patients with NB indicate a poor prognosis and are accompanied by a corresponding invasive phenotype (16). Therefore, ALK is considered to be an important oncogene in NB and a target for targeted therapy (17). A retrospective study demonstrated that the majority of adult NBs lacked MYCN amplification (18).

The prognosis of NB originating from the adrenal glands is poor, while that of NB originating from the thoracic vertebrae is more favorable, with an overall survival rate $38 \%$ higher compared with that of adrenal NB (19), which may be due to the lower risk of metastatic spread, non-amplification of the MYCN oncogene and overall favorable molecular biological characteristics. Metastasis is the most important factor in determining the prognosis of patients with NB. If recurrence or progression is suspected, comprehensive evaluation should be made in combination with CT, MRI, ${ }^{123} \mathrm{I}-\mathrm{MIBG}$ scintigraphy and positron emission tomography-CT scans (20). The prognosis of patients with NB aged $>18$ months with MYCN amplification, $1 \mathrm{p}$ deletion and $17 \mathrm{q}$ increase is poor. Preventing and reducing metastasis is the main clinical challenge in the treatment of NB (21).

The results of Wevers et al (22) showed that the high-throughput plate microfluidic model of the human blood-brain barrier may be used for drug screening. Due to the expression of human transferrin receptor on brain endothelial cells and the potential to support receptor-mediated transcellular interaction, it is of particular significance in drug targeted therapy. Identifying feasible therapeutic targets is a major challenge in the treatment of adult NB. Drug discovery is a complex and multidisciplinary process. Efficient and high-throughput screening can be used to investigate hundreds of thousands of compounds every day, which can greatly reduce the cost and time of researchers (23). In recent years, some studies have shown that the microfluidic brain microvascular chip device can directionally control cell proliferation under conditions of unidirectional flow. These cell systems exist in the vascular beds of surrounding tissues, such as kidneys or lungs, and verify the barrier function of our brain microvascular chips by measuring the permeability of fluorescent dextran and human monoclonal antibodies to further study the molecular transport mechanism of fluorescence-labeled biological products, viruses or nanoparticles across cells (24). At present, organic compounds in the human brain can be used to simulate the 

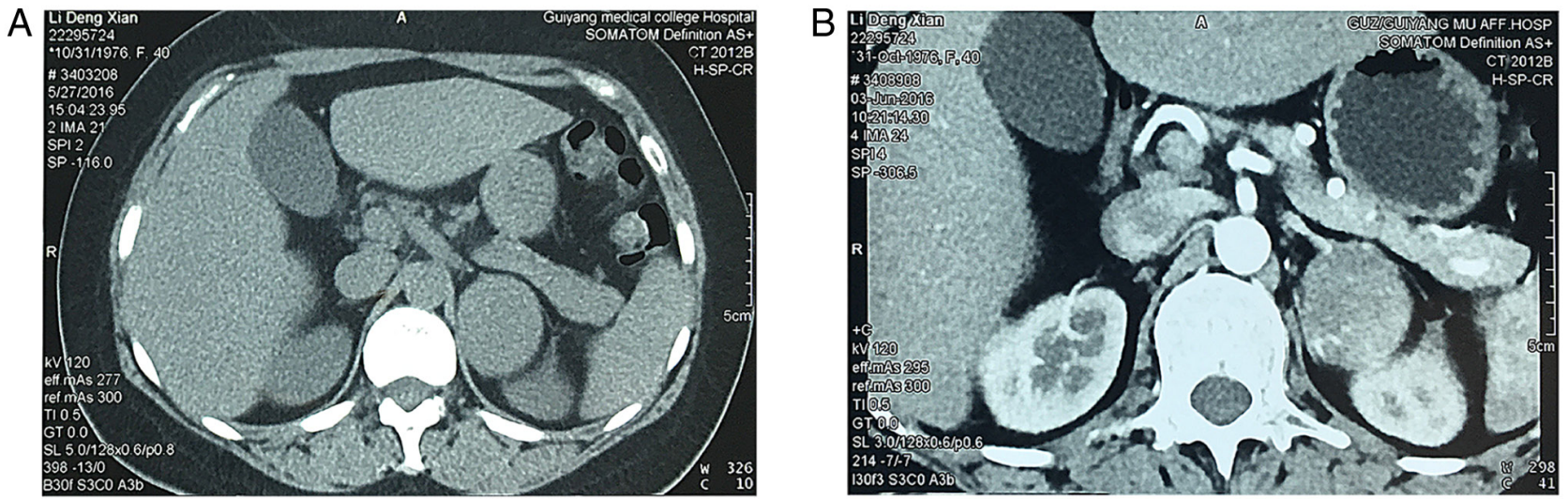

Figure 1. Abdominal CT examination. (A) An abdominal CT scan revealed a sizeable soft tissue density shadow in the left adrenal area, with uniform density and clear boundaries with surrounding tissue structures. (B) Abdominal CT with contrast enhancement revealed that the size of the tumor in the left adrenal area was $\sim 5.3 \times 5.0 \times 4.3 \mathrm{~cm}$. Following contrast-enhanced scanning, the tumor exhibited obvious inhomogeneous enhancement; the parenchyma of the tumor was obviously enhanced, while the low-density area was not enhanced, and multiple strips of blood vessels were identified in it.
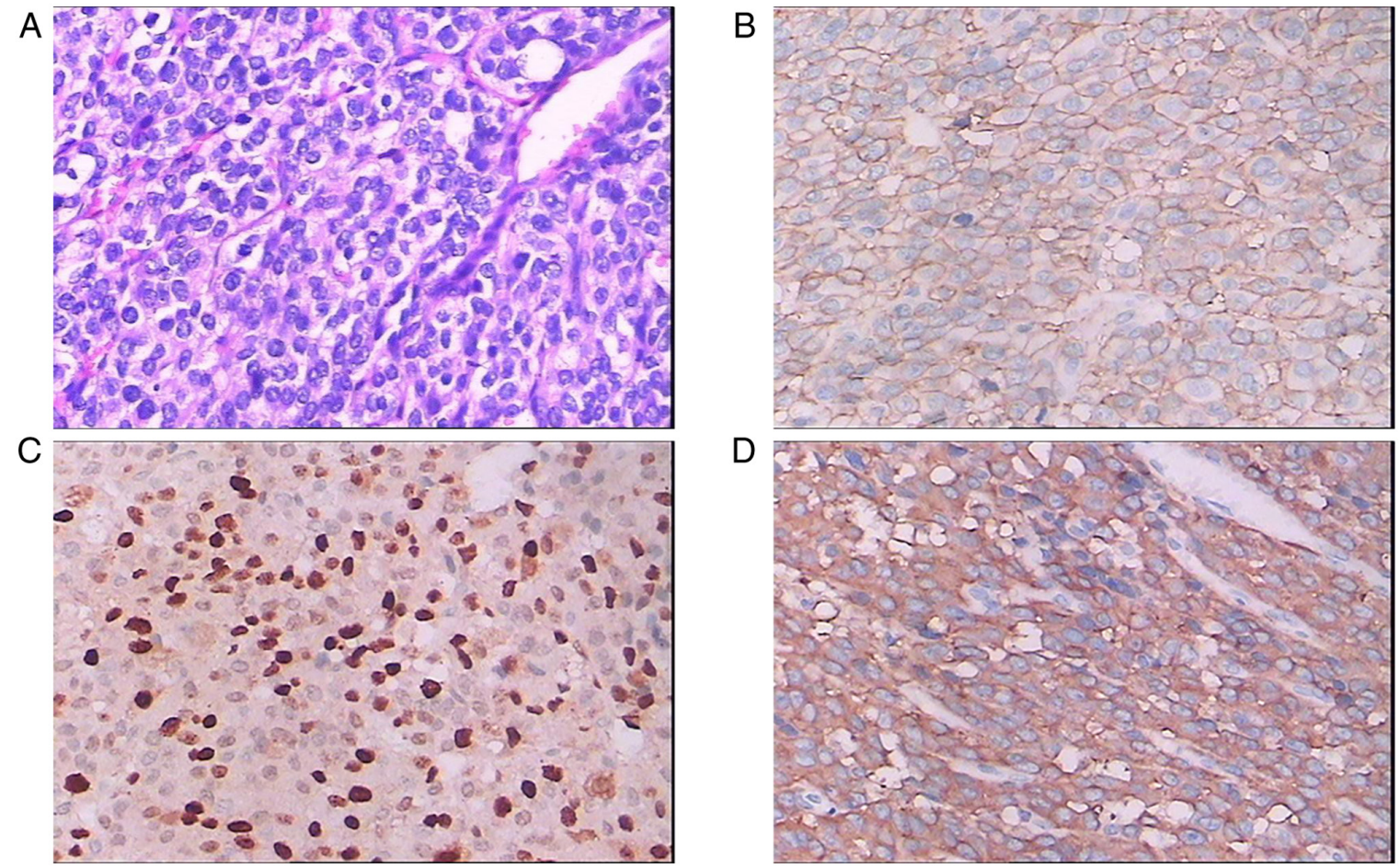

$\mathrm{D}$

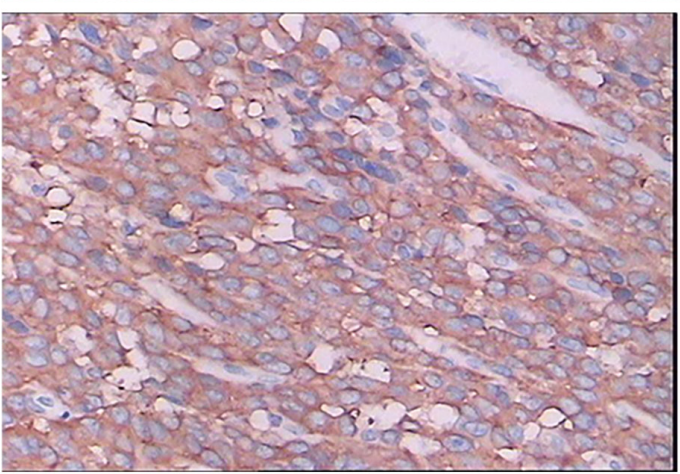

Figure 2. Histopathological examination of the resected specimen. (A) On H\&E staining of tumor sections, pathological mitotic figures were observed, and the tumor cells were arranged in nests separated by slender fibers. Immunohistochemical staining of the tumor revealed that the tumor cells were positive for (B) CD56, (C) Ki67 (80\%) and (D) synaptophysin. All magnifications, x200.

modeling, downstream analysis and application of various diseases. Human brain organic compounds provide a platform for studying the effects of substance or drug abuse on the central nervous system. The brain organic compounds of patients can be implanted with high throughput to test a large number of drugs and identify the most beneficial drugs for patients, which provides a new idea for the treatment of adrenal diseases, such as adrenal NB in adults (25).

In conclusion, adrenal NB in adults is clinically rare and highly malignant. Preoperative staging, surgical resection and postoperative adjuvant therapy can help prolong patient survival. When there is suspicion of adult NB, the feasibility of surgical treatment should be comprehensively evaluated in combination with imaging, laboratory and pathological examinations, in order to avoid a missed diagnosis or misdiagnosis.

\section{Acknowledgements}

Not applicable.

\section{Funding}

The present study was supported by the National Natural Science Foundation of China (grant no. 81660263) and Doctoral Fund of Affiliated Hospital of Guiyang Medical College, (Guiyang, Guizhou, China; grant no. C-2012-6). 


\section{Availability of data and materials}

The datasets used and/or analyzed during the current study are available from the corresponding author on reasonable request.

\section{Authors' contributions}

KT contributed to the concept and design of the article; KT, SX, WZ and BC participated in the operation; SX wrote the first draft of the article; KC and YM obtained the raw data of outpatients, such as laboratory and imaging examinations, preliminary examination of patients, and participated in the diagnosis and treatment of patients. JZ and JH provided postoperative pathological results, research direction and concepts, and provided help for the diagnosis and treatment of patients. PL and DL critically revised the manuscript as regards to the content and design of the study. All the authors confirmed the authenticity of the data.

\section{Ethics approval and consent to participate}

The present study was approved by the Ethics Committee of the Affiliated Hospital of Guizhou Medical University. The patient provided written informed consent.

\section{Patient consent for publication}

The patient consented to the publication of the case details and any associated images.

\section{Competing interests}

The authors declare that they have no competing interests.

\section{References}

1. Zhang $\mathrm{H}$ and Feng Z: Adrenal neuroblastoma in an elderly adult: A case report and review of the literature. J Med Case Rep 13: 284, 2019.

2. Bosse KR and Maris JM: Advances in the translational genomics of neuroblastoma: From improving risk stratification and revealing novel biology to identifying actionable genomic alterations. Cancer 122: 20-33, 2016

3. Conter HJ, Gopalakrishnan V, Ravi V, Ater JL, Patel S and Araujo DM: Adult versus pediatric neuroblastoma: The M.D. Anderson cancer center experience. Sarcoma 2014: 375151, 2014.

4. Bar-Sever Z, Biassoni L, Shulkin B, Kong G, Hofman MS, Lopci E, Manea I, Koziorowski J, Castellani R, Boubaker A, et al: Guidelines on nuclear medicine imaging in neuroblastoma. Eur J Nucl Med Mol Imaging 45: 2009-2024, 2018.

5. Zhuang B, Lv DK, Gao SJ and Meng JJ: Differential diagnosis of $\mathrm{CT}$ images in children with neuroblastomas and ganglioneuroblastomas. Asian Pac J Cancer Prev 15: 10509-10512, 2014.

6. D'Amuri FV, Maestroni U, Pagnini F, Russo U, Melani E, Ziglioli F, Negrini G, Cella S, Cappabianca S, Reginelli A, et al: Magnetic resonance imaging of adrenal gland: State of the art. Gland Surg 8 (Suppl 3): S223-S232, 2019.

7. Simon T: Neuroblastoma. Urologe A 44: 543-554; 555, 2005 (In German).

8. Candito M, Billaud E, Chauffert M, Cottet-Emard JM, Desmoulin D, Garnier JP, Greffe J, Hirth C, Jacob N, Millot F, et al: Biochemical diagnosis of pheochromocytoma and neuroblastomas. Ann Biol Clin (Paris) 60: 15-36, 2002 (In French).
9. Leung FY and Griffiths J: Use of plasma catecholamine values in diagnosing pheochromocytoma and neuroblastoma. Can Med Assoc J 111: 1321-1322, 1974

10. Cohn SL, Pearson AD, London WB, Monclair T, Ambros PF, Brodeur GM, Faldum A, Hero B, Iehara T, Machin D, et al: The international neuroblastoma risk group (INRG) classification system: An INRG task force report. J Clin Oncol 27: 289-297, 2009.

11. International Pediatric Endosurgery Group: IPEG guidelines for the surgical treatment of adrenal masses in children. J Laparoendosc Adv Surg Tech A 20: vii-ix, 2010.

12. Rogowitz E, Babiker HM, Kanaan M, Millius RA, Ringenberg QS and Bishop M: Neuroblastoma of the elderly, an oncologist's nightmare: Case presentation, literature review and SEER database analysis. Exp Hematol Oncol 3: 20, 2014.

13. Kushner BH, Kramer K, LaQuaglia MP, Modak S and Cheung NK: Neuroblastoma in adolescents and adults: The memorial sloan-kettering experience. Med Pediatr Oncol 41: 508-515, 2003

14. Yalçin B, Kremer LC, Caron HN and van Dalen EC: High-dose chemotherapy and autologous haematopoietic stem cell rescue for children with high-risk neuroblastoma. Cochrane Database Syst Rev: Oct 5, 2013 (Epub ahead of print). doi: 10.1002/14651858. CD006301.pub4.

15. Ertugrul MS, Nadaroglu H, Nalci OB, Hacimuftuoglu A and Alayli A: Preparation of CoS nanoparticles-cisplatin bio-conjugates and investigation of their effects on SH-SY5Y neuroblastoma cell line. Cytotechnology 72: 885-896, 2020.

16. Janoueix-Lerosey I, Lequin D, Brugières L, Ribeiro A, de Pontual L, Combaret V, Raynal V, Puisieux A, Schleiermacher G, Pierron G, et al: Somatic and germline activating mutations of the ALK kinase receptor in neuroblastoma. Nature 455: 967-970, 2008.

17. Holzhauser S, Lukoseviciute M, Papachristofi C, Vasilopoulou C Herold N, Wickström M, Kostopoulou ON and Dalianis T: Effects of PI3K and FGFR inhibitors alone and in combination, and with/without cytostatics in childhood neuroblastoma cell lines. Int J Oncol 58: 211-225, 2021.

18. Podda MG, Luksch R, Polastri D, Gandola L, Piva L, Collini P, Cefalo G, Terenziani M, Ferrari A, Casanova M, et al: Neuroblastoma in patients over 12 years old: A 20 -year experience at the istituto nazionale tumori of milan. Tumori 96: 684-689, 2010.

19. Salim A, Raitio A, Pizer B, Mullassery D and Losty PD: Neuroblastoma: The association of anatomical tumour site, molecular biology and patient outcomes. ANZ J Surg 91: 1000-1004, 2021.

20. Simon T, Hero B, Schulte JH, Deubzer H, Hundsdoerfer P, von Schweinitz D, Fuchs J, Schmidt M, Prasad V, Krug B, et al: 2017 GPOH Guidelines for diagnosis and treatment of patients with neuroblastic tumors. Klin Padiatr 229: 147-167, 2017.

21. Bhoopathi P, Pradhan AK, Bacolod MD, Emdad L, Sarkar D, Das SK and Fisher PB: Regulation of neuroblastoma migration, invasion, and in vivo metastasis by genetic and pharmacological manipulation of MDA-9/Syntenin. Oncogene 38: 6781-6793, 2019.

22. Wevers NR, Kasi DG, Gray T, Wilschut KJ, Smith B, van Vught R, Shimizu F, Sano Y, Kanda T, Marsh G, et al: A perfused human blood-brain barrier on-a-chip for high-throughput assessment of barrier function and antibody transport. Fluids Barriers CNS 15: $23,2018$.

23. Aldewachi H, Al-Zidan RN, Conner MT and Salman MM: High-Throughput screening platforms in the discovery of novel drugs for neurodegenerative diseases. Bioengineering (Basel) 8: 30, 2021.

24. Salman MM, Marsh G, Kusters I, Delincé M, Di Caprio G, Upadhyayula S, de Nola G, HuntR, Ohashi KG, Gray T, et al:Design and validation of a human brain endothelial Microvessel-on-a-Chip open microfluidic model enabling advanced optical imaging. Front Bioeng Biotechnol 8: 573775, 2020.

25. Wang $\mathrm{H}$ : Modeling neurological diseases with human brain organoids. Front Synaptic Neurosci 10: 15, 2018. 